\title{
Reduced-order LQG control of a Timoshenko beam model
}

\author{
Philipp Braun, Erwin Hernández and Dante Kalise
}

\begin{abstract}
We present a computational approach for the construction of reducedorder controllers for the Timoshenko beam model. By means of a space discretization of the Timoshenko equations, we obtain a large-scale, finite-dimensional dynamical system, for which we compute an LQG controller for closed-loop stabilization. The solutions of the algebraic Riccati equations characterizing the LQG controller are then used to construct a balancing transformation which allows the dimensional reduction of the large-scale dynamic compensator. We present numerical tests assessing the stability and performance of the proposed approach.
\end{abstract}

Keywords. Timoshenko beam, closed-loop control, model order reduction, LQG control/ balancing.

\section{Introduction and description of the problem}

The design of mechanisms for vibration control is a highly relevant topic in structural dynamics (see for instance [17] and references therein). Among the different control strategies, model-based feedback control is widely used to generate a stable closedloop that attenuates external dynamical disturbances. The underlying complexity of this optimal design problem motivates its mathematical and numerical analysis.

We approach the structural vibration problem by considering the well-acknowledged Timoshenko beam model, describing the deformation of a beam of thickness $\tau$ over a reference configuration $\Omega \times(-\tau / 2, \tau / 2)$, where $\Omega:=(0, L)$ with $L$ the length of the beam. The vibration is characterized in terms of the rotation amplitude $\theta(x, t)$ and the transverse displacement amplitude $w(x, t)$, both depending on the space variable $x$ and the time variable $t$. For a clamped beam, the vibration is governed by the 
following second-order evolution system:

$$
\begin{aligned}
& \rho S \frac{\partial^{2} w}{\partial t^{2}}=k S G\left(\frac{\partial^{2} w}{\partial x^{2}}-\frac{\partial \theta}{\partial x}\right)+\bar{u}(x, t)+f(x, t) \quad x \in \Omega, t \in \mathbb{R}_{0}^{+}, \\
& \rho I \frac{\partial^{2} \theta}{\partial t^{2}}=E I \frac{\partial^{2} \theta}{\partial x^{2}}+k S G\left(\frac{\partial w}{\partial x}-\theta\right) \quad x \in \Omega, t \in \mathbb{R}_{0}^{+}, \\
& w(0, t)=w(L, t)=\theta(0, t)=\theta(L, t)=0 \quad t \in \mathbb{R}_{0}^{+}, \\
& w(x, 0)=w_{0}(x), \frac{\partial w}{\partial x}(x, 0)=\zeta(x) \quad x \in \Omega, \\
& \theta(x, 0)=\theta_{0}(x), \frac{\partial \theta}{\partial x}(x, 0)=\eta(x) \quad x \in \Omega .
\end{aligned}
$$

The coefficients $\rho, E$, and $G$, represent the mass density, the Young modulus and, the elasticity modulus of the shear, respectively. The coefficient $k$ is a correction factor usually taken as 5/6. The parameters $S$ and $I$ represent the sectional area of the beam and the inertia moment; in this case, considered as $S=\tau^{2}$ and $I=\tau^{4} / 12$, respectively. The term $f(x, t)$ accounts for external loads and disturbances, whereas the control $\bar{u}(x, t)$ is assumed to be a constant load in space, distributed along a fixed subset $\omega_{c}$ of the domain,

$$
\bar{u}(x, t)=\chi_{\omega_{c}}(x) u(t)
$$

where $\chi_{\omega_{c}}(x)$ denotes the indicator function over $\omega_{c}$. The feedback control problem is related to the computation of $u(t)$ by means of a mapping $K \in \mathcal{L}\left(\left[L^{2}(\Omega)\right]^{4}, \mathbb{R}\right)$ acting over the current state of the system $y=(w, \theta, \dot{w}, \dot{\theta})$ (where henceforth () stands for time derivative)

$$
u(t)=-K y,
$$

such that external disturbances are compensated and the system is steered towards a certain reference trajectory. However, in a realistic setting a full knowledge of the state of the system is not available, and an additional observation equation

$$
z(t)=C y
$$

with $C \in \mathcal{L}\left(\left[L^{2}(\Omega)\right]^{4}, \mathbb{R}^{m}\right)$, is often considered. As a consequence, the feedback operator is evaluated over estimates $p$ constructed from the measurements and the knowledge of the system dynamics,

$$
u(t)=-K p .
$$

This paper concerns the study and numerical approximation of such a control loop over the infinite-dimensional dynamics defined by the Timoshenko model. The approach that we follow considers in a first step a space discretization of the dynamics leading to a large-scale dynamical system, to subsequently address the design problem at a finite-dimensional level. The theory of optimal feedback control under partial information for linear, finite-dimensional systems is well understood and dates back to the seminal work by Kalman [11]. Feedback control design for infinite-dimensional systems following the aforementioned approach have been studied in several cases, e.g., $[3,6,14]$. In particular, similar problems for the Timoshenko model have been 
studied in $[18,19,9]$. One drawback of the approach is the high complexity of the resulting controller, as the number of state space variables is directly linked to the discretization parameters used in the first step. Furthermore, the computation of the controller/observer pair involves solving Riccati equations of the same order. Instead, we propose an intermediate step of model order reduction, allowing to approximate the dynamics (and therefore the controller) by a considerably lower dimensional system. Model order reduction is a well-established technique for the simulation of control of large-scale systems [4, 7], and has been successfully applied in the control of infinite-dimensional systems $[1,5,8,12]$. In this paper we follow a linear quadratic Gaussian control/reduction approach as presented in [15], where the reduction is obtained as a by-product of the computation of the feedback control.

The paper is structured as follows. In section 2, we present the abstract, infinitedimensional setting and its numerical approximation via a locking-free finite element formulation. In section 3, we address the finite-dimensional optimal control and estimation problems by means of an LQG controller, which is then reduced using an ad-hoc balanced truncation algorithm. Finally, in section 4 we present numerical experiments illustrating the performance of the proposed approach.

\section{The abstract setting and its approximation}

In order to fit the problem within the classical settings of linear control theory, we begin by recasting eqns. (1)-(5) as a first-order evolution system. By considering the elastic operator

$$
\mathcal{A}(w, \theta):=-\left[\begin{array}{cc}
\frac{k G}{\rho} \frac{\partial^{2}}{\partial x^{2}} & -\frac{k G}{\rho} \frac{\partial}{\partial x} \\
\frac{k A G}{\rho I} \frac{\partial}{\partial x} & \frac{E}{I} \frac{\partial^{2}}{\partial x^{2}}-\frac{k A G}{\rho I}
\end{array}\right]\left[\begin{array}{l}
w \\
\theta
\end{array}\right], \quad D(\mathcal{A})=\left[H^{2}(\Omega) \cap H_{0}^{1}(\Omega)\right]^{2},
$$

we can define the augmented operators $(A, B)$

$$
A:=\left[\begin{array}{cc}
0 & \mathcal{I} \\
-\mathcal{A} & -\mathcal{D}(\mathcal{A})
\end{array}\right], \quad B:=\left[\begin{array}{c}
0 \\
0 \\
\chi_{\omega_{c}}(x) \\
0
\end{array}\right],
$$

where $\mathcal{I}$ corresponds to the identity operator. For sake of simplicity, henceforth we shall neglect the existence of disturbances $(f \equiv 0)$, and thus reduce the problem to the implementation of an optimal feedback regulation under partial state observation. With no additional difficulties, this can be modified in order to include the effect of noise. We have introduced a damping operator $\mathcal{D}(\mathcal{A})$, which in our case will be set as $\mathcal{D}(\mathcal{A})=\alpha \mathcal{A}, \alpha>0$, as discussed in [13, Chapter 5]. We can now represent the Timoshenko model as the first-order linear system

$$
\begin{aligned}
& \dot{y}(x, t)=A y+B u, \\
& y(x, 0)=y_{0}(x)
\end{aligned}
$$


acting over the state $y=(w, \theta, \dot{w}, \dot{\theta})^{T}$. We shall also consider the existence of an observation equation

$$
z=C(y)
$$

where the observation operator $\mathcal{C} \in \mathcal{L}\left(\left[L^{2}(\Omega)\right]^{4} R^{m}\right)$ will be defined as

$$
C(y)(t):=\left(\frac{1}{\left|\omega_{1}\right|} \int_{\omega_{1}} w(x, t) d x, \ldots, \frac{1}{\left|\omega_{m}\right|} \int_{\omega_{m}} w(x, t) d x\right),
$$

where $\left\{\omega_{i}\right\}_{i=1}^{m}$ are disjoint, non-empty subsets of $\Omega$. Having defined the abstract state space representation (6)-(8), we now turn our attention to the control design problem. We consider the state space $Y=\left[H_{0}^{1}(\Omega)\right]^{2} \times\left[L^{2}(\Omega)\right]^{2}$ such that $D(A) \subset Y$, a control space $U=\mathbb{R}$, an observation space $Z=\mathbb{R}^{m}$, and a cost functional

$$
J(y, u)=\frac{1}{2} \int_{0}^{\infty}\|z\|_{Z}^{2}+\|u\|_{U}^{2} d t .
$$

The corresponding optimal control problem reads

$$
\min _{u \in L^{2}([0, \infty) ; \mathbb{R})} J(y(u), u)
$$

subject to system dynamics and observations (6)-(8). We present some relevant results for this problem concerning the well-posedness of the optimal control problem (10). For more details concerning the abstract problem we refer to [9].

Proposition 2.1. A is the infinitesimal generator of a strongly continuous, analytic semigroup $e^{A t}$, on $Y$.

Proposition 2.2. (Finite cost condition) For every initial condition $y_{0} \in Y$, there exists $u \in L^{2}([0, \infty) ; \mathbb{R})$ such that $J(y, u)<\infty$.

Note that due to the inclusion of a damping term generates an exponential decay of the uncontrolled system, and therefore $J(y, u)<\infty$. Exponential stabilizability and detectability follow trivially $([13])$.

Theorem 2.3 ([9]). For each initial condition $y_{0} \in Y$, there exists a unique optimal pair $\left(u^{*}, y^{*}\right)$ of the abstract optimal control problem subject to the system dynamics (6).

Formally, the solution of the optimal control problem (10) with partial observation (the LQG control problem) is expressed as a dynamic compensator of the form

$$
\begin{aligned}
& \dot{y}=A y+B u, \\
& z=C y, \\
& \dot{p}=(A-B K) p+F(z-C p), \\
& u=-K p,
\end{aligned}
$$

with

$$
K=B^{*} \mathcal{P}, \quad F=\mathcal{Q} C^{*},
$$


where the operators $\mathcal{P}$ and $\mathcal{Q} \in \mathcal{L}(Y, Y)$ are the unique non-negative self-adjoint operators satisfying the following operator Riccati equations

$$
\begin{aligned}
& A^{*} \mathcal{P}+\mathcal{P} A-\mathcal{P} B B^{*} \mathcal{P}+C^{*} C=0, \\
& A \mathcal{Q}+\mathcal{Q} A^{*}-\mathcal{Q} C^{*} C \mathcal{Q}+B B^{*}=0 .
\end{aligned}
$$

Note that these are abstract operator equations over an infinite-dimensional state space. In what follows, we shall generate an approximating sequence of finite-dimensional state-space representations $\left(A_{h}, B_{h}, C_{h}\right)$, leading to approximate solutions $\left(\mathcal{P}_{h}, \mathcal{Q}_{h}\right)$ which converge to the solution of this abstract problem when $h$ goes to 0 . For this purpose, the first step is to consider a discretization in space of the system leading to a finite-dimensional representation of the dynamics. In this context, the application of standard finite elements or finite difference schemes is usually sufficient to generate a theoretically and computationally convergent sequence of problems (see for instance, the series of examples in [13, Chapter 5]). However, it is well-known that the application of standard finite element approximations to the Timoshenko model leads to the so-called locking-phenomenon, which produces unsatisfactory results when the beam tichkness parameter $\tau$ is decreased [2]. In this latter reference, a locking-free mixed finite element formulation is proposed. The application of this technique for the control problem (10) has been presented in [9], and we adopt a similar approach. We briefly recall the most relevant steps in the locking-free discretization.

Let us consider a finite-dimensional approximating subspace $\mathcal{V}_{h} \subset H_{0}^{1}(\Omega)$, to be a piecewise linear finite element space. For this reason, we consider a family $\left\{\mathcal{T}_{h}\right\}$ of regular partitions of the interval $\Omega$ :

$$
\mathcal{T}_{h}: 0=x_{0}<x_{1}<\cdots<x_{n}=L,
$$

with mesh size $h:=L / n$. The subspace $\mathcal{V}_{h}$ can be written as

$$
\mathcal{V}_{h}:=\left\{v \in H_{0}^{1}(\Omega):\left.v\right|_{\left[x_{j-1}-x_{j}\right]} \in \mathbb{P}_{1}, j=1, \ldots, n\right\} \subset H_{0}^{1}(\Omega) .
$$

Let $\mathcal{V}_{h_{1}}$ consists of the elements of $\mathcal{V}_{h}$ and equipped with the $H^{1}(\Omega)$ seminorm and let $\mathcal{V}_{h_{2}}$ consists of the elements of $\mathcal{V}_{h}$ equipped with the $L^{2}(\Omega)$ norm. We set $V_{h}=$ $\mathcal{V}_{h_{1}}^{2} \times \mathcal{V}_{h_{2}}^{2}$. We denote by $P_{h}$ the orthogonal projection from $\left[L^{2}(\Omega)\right]^{4}$ onto $V_{h}$, i.e., $P_{h}:=\pi_{h} \mathbf{I}_{4}$, where $\mathbf{I}_{4}$ denotes the identity matrix in the square matrices of size 4 , and $\pi_{h}$ represents the orthogonal projection from $L^{2}(\Omega)$ onto $\mathcal{V}_{h}$. The subspace $\mathcal{V}_{h}$ satisfies the approximation property

$$
\left\|\pi_{h} v-v\right\|_{\left.H^{l} \Omega\right)} \leq C h^{s-l}\|v\|_{H^{s}(\Omega)}, \quad \forall v \in H^{s}(\Omega) \cap H_{0}^{1}(\Omega), \quad 0 \leq l \leq s \leq 2 .
$$

To define a locking-free scheme for the approximation of the Timoshenko model, we consider the following discrete space:

$$
\mathcal{W}_{h}:=\left\{\frac{d v}{d x}+c, v \in \mathcal{V}_{h}, c \in \mathbb{R}\right\} \subset L^{2}(\Omega) .
$$

The Galerkin approximation of the operator $A$ is defined upon the following weighted $L^{2}(\Omega) \times L^{2}(\Omega)$ inner product:

$$
\langle(\eta, \varsigma),(v, \beta)\rangle_{\tau}=(\eta, v)+\frac{\tau^{2}}{12}(\varsigma, \beta) .
$$


The Galerkin approximation of the operator $A$ on $V_{h}$ is defined as follows:

$$
A_{h}=\left[\begin{array}{cc}
0 & \Pi_{h} \\
-\mathcal{A}_{h} & -\alpha \mathcal{A}_{h}
\end{array}\right]: V_{h} \rightarrow V_{h}, \quad \Pi_{h}=\left[\begin{array}{cc}
\pi_{h} & 0 \\
0 & \pi_{h}
\end{array}\right],
$$

where $\mathcal{A}_{h}$ denotes the locking-free approximation of the operator $\mathcal{A}$, defined by means of the bilinear form

$$
\begin{aligned}
\left\langle\mathcal{A}_{h}\left(w_{\tau h}, \theta_{\tau h}\right),\left(v_{h}, \beta_{h}\right)\right\rangle_{\tau}= & \frac{E}{12 \hat{\rho}} \int_{\Omega} \frac{d \theta_{\tau h}}{d x} \frac{d \beta_{h}}{d x} d x \\
& +\frac{\kappa}{\tau^{2} \hat{\rho}} \int_{\Omega} \pi_{h}^{0}\left(\frac{d w_{\tau h}}{d x}-\theta_{\tau h}\right) \pi_{h}^{0}\left(\frac{d v_{h}}{d x}-\beta\right) d x,
\end{aligned}
$$

for all $\left(w_{\tau h}, \theta_{\tau h}\right),\left(v_{h}, \beta_{h}\right) \in \mathcal{V}_{h}^{2}$, where $\pi_{h}^{0}$ denotes the projection from $L^{2}(\Omega)$ onto $\mathcal{W}_{h}$. Moreover, we have considered a rescaling in the density of the material, $\rho=\hat{\rho} \tau^{2}$. For more details concerning the locking-free discretization of the Timoshenko beam model in the context of optimal control, we refer to [10]. The approximation of the operator $B$ is given by

$$
B_{h} u:=P_{h} B u=\left[\begin{array}{c}
0 \\
0 \\
\pi_{h} \chi_{\omega_{c}} u(t) \\
0
\end{array}\right]=\left[\begin{array}{c}
0 \\
0 \\
\chi_{\omega_{c}} u(t) \\
0
\end{array}\right]: \mathbb{R} \rightarrow V_{h} .
$$

Finally, the obervation operator $C$ is approximated as

$$
C_{h}(y)(t):=\left(\frac{1}{\left|\omega_{1}\right|} \int_{\omega_{1}} \pi_{h} w(x, t) d x, \ldots, \frac{1}{\left|\omega_{m}\right|} \int_{\omega_{m}} \pi_{h} w(x, t) d x\right) .
$$

This locking-free Galerkin approximation generates a finite-dimensional sequence state space representations $\left(A_{h}, B_{h}, C_{h}\right)$ for which the solution the optimal control problem converge to the solution of the abstract problem (10). This has been extensively discussed in previous works $[3,13,9]$.

\section{LQG balancing}

The key point in the synthesis of the approximate LQG control is the solution of the Riccati equations (16)-(17) for the system $\left(A_{h}, B_{h}, C_{h}\right)$, which is a computationally demanding task for large-scale systems such as those generated in this semi-discrete setting. Assuming that it is possible to solve this problem in a rather expensive offline phase, the resulting dynamic compensator (13)-(14) is of the same dimensions of the state space representation. This represents a prohibitive limitation for the implementation of an online controller, thus making necessary to obtain a reducedorder controller. For this purpose we apply an LQG-balanced truncation algorithm as in [15], based on the balancing of the operators $\mathcal{P}$ and $\mathcal{Q}$. We look for a similarity transformation such that $\mathcal{P}$ and $\mathcal{Q}$ are transformed into a diagonal matrix $\mathbb{D}=\left(\lambda_{1}, \ldots, \lambda_{n}\right)$ of LQG-characteristic values, $\lambda_{1} \geq \lambda_{2} \geq \ldots>0$. Then, we apply this transformation either to the state space representation of the system or to the compensator to obtain a hierarchical representation in balanced coordinates. The 
balanced representation is then truncated up to the first $r$ coordinates, yielding a reduced system $\left(A_{h}^{r}, B_{h}^{r}, C_{h}^{r}\right)$. For the details concerning the efficient solution of the LQG balancing problem, we refer the reader to [5]. In this context, the design of a reduced-order controller can be performed in two ways. The first alternative is to consider an LQG-balanced truncation of the dynamics, and then solve the LQG control problem for the reduced dynamics, leading to a reduced-order compensator. Instead we opt for solving the LQG problem over the full dynamics, and as a by product of the solution of the Riccati equations, obtain the balancing transformation in order to truncate the full-order compensator. The following result provides an error bound for the LQG-balanced truncation algorithm.

Theorem 3.1. When the $L Q G$-balanced truncation algorithm is applied over a finitedimensional system $(A, B, C)$, the following error bound for the output $z_{r}$ of the reduced model $\left(A^{r}, B^{r}, C^{r}\right)$ holds:

$$
\left\|z-z_{r}\right\|_{L^{2}\left(0, T ; \mathbb{R}^{m}\right)} \leq 2\|u\|_{L^{2}(0, T ; \mathbb{R})} \sum_{i=r+1}^{n} \frac{\lambda_{j}}{\sqrt{1+\lambda_{j}^{2}}} .
$$

\section{Numerical tests}

We present two numerical tests highlighting the main features of the proposed approach. In this tests, we discretize the Timoshenko beam model by means of the locking-free finite element discretization with mesh parameter $h$ as described in section 2 , leading to a finite-dimensional state-space representation $\left(A_{h}, B_{h}, C_{h}\right)$. For this system, we solve the Riccati equations (16)-(17), and construct the balancing transformation for the reduced-order compensator by means of the LYAPACK MATLAB toolbox [16]. We consider two tests with $\tau=0.1$ (Test 1) and $\tau=0.01$ (Test 2), the remaining parameters are set $\alpha=10^{-6}, L=1, h=0.01$, $E=2.1 \times 10^{11}[\mathrm{~Pa}], \rho=7.8 \times 10^{3}\left[\mathrm{Kg} / \mathrm{m}^{3}\right], G=E / 2.6$, a distributed control along $\omega_{c}=[0.3,0.6]$, and 10 uniformly distributed observations of length $h / 2$. Initial conditions are $w(x, t)=\sin (\pi x)$ and zero for the remaining state variables. The dynamical behavior of the uncontrolled system is obtained with a trapezoidal implicit scheme with time step $\Delta t=h / 50$, and is it shown for the two test cases in Figure 1 . It can be seen that despite the small damping factor, it is enough to generate a strong dissipative effect for thick beams, whereas in the thin case the model it produces an almost undamped output. A direct consequence can be seen in the decay of the LQGcharacteristic values, when for $\tau=0.1$ its strong decay suggest a good approximation of the model with a few number of balanced states (which is usual for parabolic-like dynamical systems). In the case $\tau=0.01$ which recovers the hyperbolic nature of the undamped system, the decay is very slow and a large number of balanced states will be required to guarantee stabilization of the full-order model.

Test 1 . In this thick beam test $\tau=0.1$ our purpose is to validate the reduced-order controller and the observer design. As the strong decay of the LQG-chracteristic values suggests, in this case it is enough to take a reduced order model of 2 states to generate a stabilizing compensator for the full-order model. Stabilization of the state variables and the evolution of the observer error are shown in Figure 2. The 

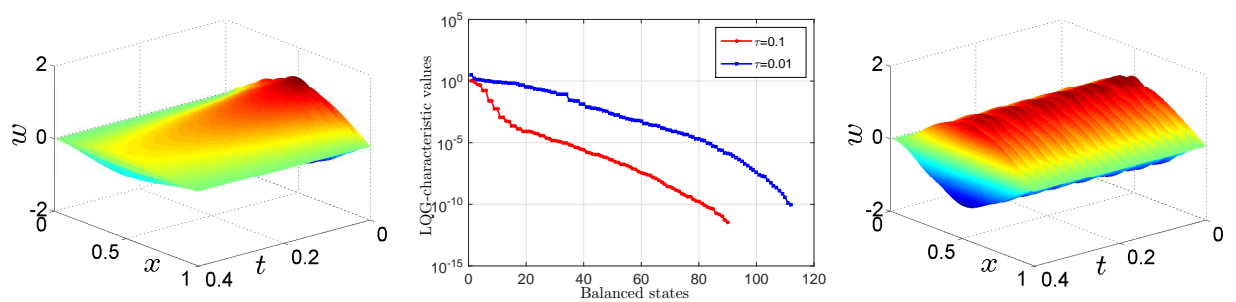

FiguRE 1. Uncontrolled state $w$ for thickness $\tau=0.1$ (left) and $\tau=$ 0.01 (right). The decay of the LQG-characteristic values (middle) provides a guideline for dimensional reduction.

difference between the stabilization rate of the state variables and the decay of the observer error is due to the scaling of the velocity variables $\dot{w}$ and $\dot{\theta}$.
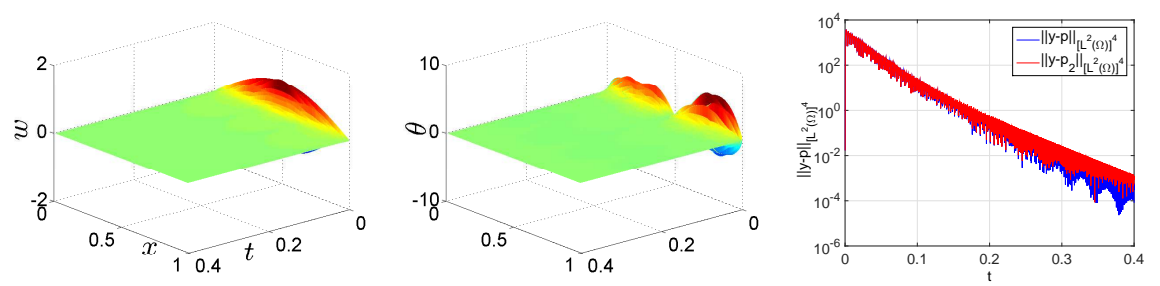

Figure 2. Test 1. Evolution of the controlled states $w$ (left) and $\theta$ (middle) with a reduced-order control of dimension 2. In the right, we see the evolution of the observer error for the full-order estimate $p$ and the reduced-order observer $p_{r}$.

Test 2. In the thin beam case $\tau=0.01$, we study the limitations of the proposed approach. As it has been observed in Figure 1, the damping effect in this case is negligible and the low dissipation translates into a very slow decay of the LQG-characteristic values. In fact, for less than 20 reduced states, the reduced-order compensator is not able to consistently stabilize the full-order dynamics. The first satisfactory results are obtained with $r=22$ as shown in Figure 3. Although this is a radically different scenario if compared with Test 1 , it must be noted that the reduced order controller for $r=20$ has an efficiency factor of $20 \times$ with respect to the the full-order compensator. Figure 4 indicates that with $r=40$ states, i.e. with an efficiency factor of $10 \times$, it is possible to replicate the full order-dynamics and controller in a very accurate way.

Concluding remarks. We have discussed the application of a reduced-order control algorithm for the stabilization of the Timoshenko beam model. As a by product of the design of an LQG controller, is it possible to obtain a balancing transformation for the dimensional reduction of the dynamic compensator. In this context, a generic requirement for the approach to be successful is the fast decay of the LQG-characteristic 

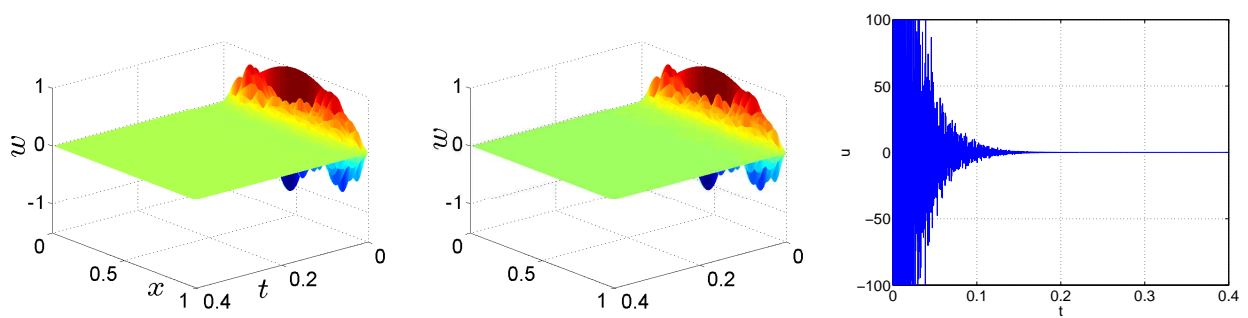

Figure 3. Test 2. Full order controlled $w$ (left), reduced-order $(r=$ 22 ) controlled $w$ (middle) and control signal $u(t)$ (left).
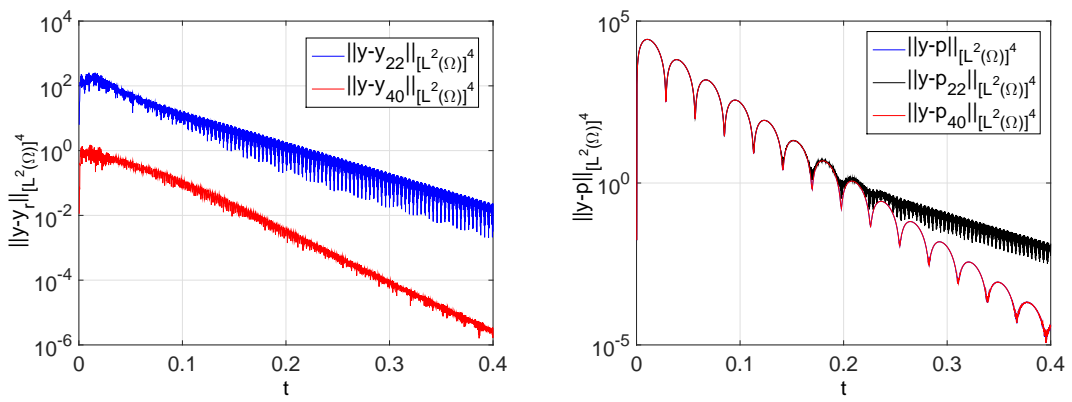

Figure 4. Test 2. Evolution of the difference between the state with full-order control $y$ and the state with reduced-order control $y_{r}$ (left). Difference between the full-order controlled state $y$ and the reduced-order state estimator $p_{r}$ extended to the full space (right).

values. For systems governed by partial differential equations, this relates to the existence of dissipative effects within the system. Therefore in our case the possibility of synthesizing a low-complexity controller is conditioned to the interplay between the structural damping term and the model parameters such as the thickness of the beam. A relevant question that remains open is to determine a lower bound for the number of reduced states which are necessary for a reduced compensator to guarantee the stabilization of the full-order dynamics. This relates to the $\mathcal{H}_{\infty}$ control and reduction problem. In [15], the authors present a sufficiency test for determining the number of reduced states for full-order stabilization. However, in our experience these estimates are not optimal, and therefore we aim at improving the aforementioned bounds for specific models arising in structural vibration.

Acknowledgments. The authors wish to thank Lars Grüne for fruitful discussions which motivated this work. The second author was supported by FONDECYT under Grant No. 1140392, BASAL Project (CMM, UChile), and CONICYT Anillo ACT1106. 


\section{References}

[1] A. Alla, M. Falcone and D. Kalise. An efficient policy iteration algorithm for dynamic programming equations, SIAM J. Sci. Comput., 37(1), 181-200, 2015.

[2] D.N. Arnold. Discretization by finite element of a model dependent parameter problem, Numer. Math. 37 (1981), 405-421.

[3] H.T. Banks and K. Kunisch. The linear regulator problem for parabolic systems, SIAM J. Control Optim. 22(5) (1984), 684-698.

[4] P. Benner. Solving large-scale control problems, IEEE Control Syst. Mag. 14(1) (2004), 44-59.

[5] P. Benner. Balancing-Related Model Reduction for Parabolic Control Systems, Control of Systems Governed by Partial Differential Equations, IFAC, Volume 1, Part 1, 257$262,2013$.

[6] T. Breiten and K. Kunisch. Compensator design for the monodomain equations, preprint, http://math.uni-graz.at/kunisch/papers/KK_284.pdf.

[7] S. Gugercin and A. Antoulas. A survey of model reduction by balanced truncation and some new results, Int. J. Control 77(8) (2004), 748-766.

[8] D. Kalise and A. Kröner. Reduced-order minimum time control of advection-reactiondiffusion systems via dynamic programming, Proceedings of the 21st International Symposium on Mathematical Theory of Networks and Systems, 1196-1202 (2014).

[9] E. Hernández, D. Kalise and E. Otárola. A locking-free scheme for the LQR control of a Timoshenko beam, J. Comput. Appl. Math. 235(5)(2011), 1383-1393.

[10] E. Hernández and E. Otárola. A locking-free FEM in active vibration control of a Timoshenko beam, SIAM J. Numer. Anal. 47 (2009), 2432-2454.

[11] R.E. Kalman. Contributions to the theory of optimal control, Bol. Soc. Mat. Mex. 5 (1960), 102-119.

[12] K. Kunisch, S. Volkwein, L. Xie. HJB-POD Based Feedback Design for the Optimal Control of Evolution Problems, SIAM J. on Applied Dynamical Systems 4 (2004), 701722 .

[13] I. Lasiecka and R. Triggiani. Control theory for partial differential equations: continuous and approximations theories, Encyclopedia of mathematics and its applications 74, Cambridge University Press, 2000.

[14] K.A. Morris. Design of finite-dimensional controllers for infinite-dimensional systems by approximation, J. Math. Systems Estim. Control 6(2)(1996), 151-180.

[15] D. Mustafa and K. Glover. Controller reduction by H-infinity-balanced truncation, IEEE Trans. Automat. Control 36(6) (1991), 668-682.

[16] T. Penzl. LYAPACK - Users' Guide (Version 1.0), available at: ftp://164.41.45.3/ $\mathrm{pub/netlib/lyapack/guide.pdf.}$

[17] A. Preumont. Vibration Control of Active Structures, 3rd ed., Solid Mechanics and its Applications 179, Springer, 2011.

[18] M. Tadi. Optimal infinite-dimensional estimator and compensator for a Timoshenko Beam, Computers Math. Applic. 27(6) (1994), 19-32.

[19] M. Tadi. Computational algorithm for controlling a Timoshenko beam, Comput. Methods Appl. Mech. Engrg. 153 (1998), 153-165. 


\section{Philipp Braun}

University of Bayreuth

Chair of Applied Mathematics

Universitätsstraße 30

95440 Bayreuth, Germany

e-mail: philipp.braun@uni-bayreuth.de

Erwin Hernández

Technical University Federico Santa María

Department of Mathematics

Avenida España 1680

Valparaíso, Chile

e-mail: erwin.hernandez@usm.cl

Dante Kalise

Radon Institute for Computational and Applied Mathematics (RICAM)

Austrian Academy of Sciences

Altenbergerstraße 69

A-4040 Linz, Austria

e-mail: dante.kalise@oeaw.ac.at 